\title{
Calculadoras de riesgo cardiovascular como estrategia preventiva de eventos isquémicos en la población de Latinoamérica
}

DOI 10.5377/alerta.v4i1.10269

Reyna de la Paz Orellana Flores ${ }^{1}$

Iris Gabriela Portillo Benítez ${ }^{2}$

Mónica Alejandra Villarroel Martínez

Facultad de Ciencias de la Salud Dr. Luis Edmundo Vásquez, Universidad Dr. José Matías Delgado 1,2,3, Antiguo Cuscatlán, El Salvador

${ }^{*}$ Correspondencia

$\square$ irisgabriela4408@gmail.com

1. (1) 0000-0002-0044-6657

2. (1) 0000-0002-7284-8161

3. (1) 0000-0001-5803-1932

a

Cardiovascular Risk calculators as preventive strategy of ischemic events in the Latin American population

\section{Citación recomendada:} Orellana Flores RP, Portillo Benítez IG, Villarroel Martínez MA. Calculadoras de riesgo cardiovascular como estrategia preventiva de eventos isquémicos en la población de Latinoamérica. Alerta. 2021:4(1):40-48. DOI 10.5377/ alerta.v4i1.10269.

Recibido:

8 de octubre de 2020

Aceptado:

7 de enero de 2021

Publicado:

22 de enero 2021

Contribución de autoría: $\mathrm{RPOF}^{1}$, IGPB ${ }^{2}, \mathrm{MAVM}^{3}$.

Búsqueda de material bibliográfico, orden y corrección de Manuscrito.

\section{Conflicto de intereses:}

Las autoras declaran no tener ningún conflicto de interés.

\section{Resumen}

A mitad del siglo XX surge un nuevo paradigma enfocado en la prevención y no únicamente en el manejo de pacientes con enfermedades cardiovasculares. Este enfoque busca identificar indicadores de riesgo involucrados en el desarrollo de dichas patologías para generar intervenciones preventivas. En la actualidad se cuenta con múltiples escalas de riesgo para calcular la posibilidad de eventos cardiovasculares mayores en un tiempo determinado. En esta revisión bibliográfica se presenta una serie de escalas y calculadoras de riesgo utilizadas con frecuencia, y se describen sus diferentes cualidades, beneficios, oportunidades, fortalezas y debilidades. Se revisó la evidencia de las siguientes herramientas: Framingham, QRISK3 (algoritmo QRESEARCH de riesgo cardiovascular), American Heart Association, Organización Mundial de la Salud, Systematic Coronary Risk Evaluation y Gaziano. Es fundamental investigar la aplicación de estas calculadoras de forma rutinaria en la población de Latinoamérica para determinar cuál herramienta es la más adecuada.

Palabras clave

Infarto del miocardio, enfermedades cardiovasculares, síndrome metabólico, factores de riesgo, atención primaria.

\section{Abstract}

In the middle of the 20th century, a new paradigm emerged focusing on prevention and not just on management of patients with cardiovascular diseases. This approach seeks to identify risk indicators involved in the development of these pathologies, to design preventive interventions. Currently there are multiple risk scales to calculate the possibility of major cardiovascular events in a given time. In this bibliographic review, a series of frequently used scales are presented, describing their different qualities, benefits, opportunities, strengths, and weaknesses. Evidence from the following tools have been reviewed: Framingham, QRISK3 (QRESEARCH cardiovascular risk algorithm), American Heart Association, World Health Organization, Systematic Coronary Risk Evaluation and Gaziano. It is essential to investigate the application of this calculators in a routinely manner to determine which tool may be more appropriate according to the characteristics of the Latin American population.

Keywords

Myocardial infarction, cardiovascular disease, metabolic syndrome, risk factors, primary health care.

\section{Introducción}

Las enfermedades cardiovasculares (ECV) son las patologías que afectan al corazón y a los vasos sanguíneos; se encuentran entre las principales causas de morbimortalidad y alta probabilidad de discapacidad ${ }^{1,2}$. Las ECV son la principal causa de mortalidad a nivel mundial, sobrepasando las 200 muertes por cada 100000 habitantes, correspondiendo 
13,13\% al infarto miocárdico, 2,24 \% a hipertensión arterial y a otra patología isquémica un $2,08 \%{ }^{3}$.

El riesgo cardiovascular se define como «la probabilidad de una persona de morir o sufrir eventos mayores cardiocirculatorios como infarto agudo al miocardio, tromboembolismos e incluso la muerte en un periodo determinado de tiempo» ${ }^{4,5}$. Se estima que alrededor de 23,3 millones de personas morirán a causa de ECV en 2030, y se espera que en el futuro sigan siendo la principal causa de muerte ${ }^{6,7}$. El estudio Framingham puso en perspectiva la presencia de factores de riesgo cardiovascular y se han hecho múltiples intentos por desarrollar calculadoras de alto valor predictivo, pero con el grado de sencillez adecuado para una rápida implementación a gran escala-11.

Latinoamérica no es la excepción en cuanto a elevadas tasas de morbimortalidad por ECV. Pocos han tenido el interés de investigar el uso de las calculadoras y quienes lo han realizado no presentan evidencia concreta sobre alguna que tenga aplicabilidad y sea extrapolable a la población latinoamericana con mejores resultados debido a limitaciones, como costos elevados de atención y la necesidad de realizar exámenes de laboratorio ${ }^{12}$.

La evaluación del riesgo cardiovascular, sobre todo en el primer nivel de atención de los países en vías de desarrollo, genera un aporte invaluable para el manejo del paciente, sentando las directrices del seguimiento y la intensidad de las intervenciones, con un enfoque preventivo de los eventos ${ }^{13-15}$.

Por tanto, en este trabajo se pretende analizar diferentes tablas de predicción de riesgo cardiovasculares y discutir su aplicabilidad en la población latinoamericana. Para tal efecto se realizó una revisión bibliográfica de artículos originales y de revisión con una vigencia de publicación no mayor de 5 años. Se consultaron bases de datos como: SciELO, Google Académico, HINARI y PubMed, en idioma español e inglés, haciendo uso de operadores booleanos como AND, OR y NOT: Riesgo cardiovascular AND Infarto Agudo al Miocardio, Cardiopatía Isquémica NOT Síndrome Metabólico, Riesgo cardiovascular elevado OR Diabetes Mellitus. Se utilizaron los siguientes descriptores claves: calculadoras de riesgo cardiovascular, riesgo cardiovascular, enfermedad cardiovascular, factores de riesgo cardiovascular.

\section{Desarrollo de la temática}

\section{Escala Framingham}

El estudio Framingham comenzó en 1948 con el grupo original (cohorte original) ${ }^{16}$. En 1971 se añadió a los hijos de la cohorte original (cohorte offspring) ante la necesidad de un estudio prospectivo en adultos jóvenes ${ }^{17}$. Posteriormente, en 2002, el estudio reclutó una tercera generación de participantes; que permitiría aportar información sobre los factores fenotípicos y genotípicos relacionados al riesgo cardiovascular ${ }^{18}$. En 1994 se agregó un grupo multicultural (Omni) ante la necesidad de reflejar la diversidad étnica, seguido de un segundo grupo multicultural en 2003.

El estudio de Framingham proporciona dos opciones para valorar el riesgo cardiovascular, calculando tal riesgo en los próximos 10 o 30 años ${ }^{19,20}$. La calculadora para 10 años se utiliza en pacientes entre las edades de 30 a 74 años, sin historial de enfermedad cardiovascular; utiliza los predictores: edad, diabetes, condición de fumador, colesterol total, colesterol Lipoproteínas de alta densidad (HDL por sus siglas en inglés), índice de masa corporal (IMC) y si el paciente en estudio se encuentra en tratamiento para manejo de presión arterial sistólica.

La calculadora, que predice el riesgo cardiovascular para los siguientes 30 años, está diseñada para pacientes entre las edades de 20 a 59 años, sin antecedentes de enfermedad cardiovascular o cáncer. Utiliza los predictores: sexo, edad, presión arterial sistólica, uso o no de antihipertensivos, condición de fumador, diabetes mellitus, colesterol total, colesterol HDL e IMC.

\section{Algoritmo de predicción de riesgo cardiovascular QRISK3}

El primer modelo de QRISK fue publicado en 2007, en busca de una herramienta enfocada para la población europea, que permitiera estimar el riesgo cardiovascular a 10 años en pacientes de atención primaria. El modelo fue actualizado en 2008 (QRISK2) ${ }^{21}$. En el año 2017 se publicó la versión actualizada QRISK322-24.

El algoritmo QRISK2 valora edad, sexo, origen étnico, presión arterial sistólica, IMC, cociente colesterol total/HDL, tabaquismo, antecedente familiar de enfermedad coronaria en parientes de primer grado menores de 60 años, tratamiento con antihipertensivos, artritis reumatoidea, fibrilación atrial, enfermedad renal crónica (estadio 4 y 5), síndrome nefrótico, glomerulonefritis crónica, pielonefritis crónica, pacientes en diálisis renal y pacientes con trasplante renal ${ }^{2}$. QRISK3 cuantifica el riesgo cardiovascular en personas entre la edad de 25 a 84 años, incluyendo otros factores de riesgo para aumentar la capacidad predictiva. 


\section{Calculadora de ACC/AHA (2013)}

En 2013, la American College of Cardiology y la American Heart Association (ACC/AHA) desarrollaron una calculadora de riesgo cardiovascular que basa su calculo a partir de datos de población blanca y afroamericana; predice el riesgo a 10 años de sufrir complicaciones de enfermedad cardiovascular aterosclerótica en pacientes que no presentan enfermedad previa e identifica los candidatos óptimos para recibir terapia con estatinas cuando presentan un resultado de riesgo cardiovascular elevado ${ }^{25-27}$.

Esta calculadora pondera el riesgo a 10 años de sufrir una enfermedad cardiovascular aterosclerótica en pacientes que no presentan enfermedad previa e identifica los candidatos óptimos para recibir terapia con estatinas cuando presentan un resultado de riesgo cardiovascular elevado.

\section{Calculadora de OMS}

Se ha propuesto el uso de las tablas de predicción de la Organización Mundial de la Salud (OMS). Estas incluyen datos sobre la prevalencia de factores de riesgo y el riesgo relativo según la subregión epidemiológica ${ }^{28}$. Es una calculadora que ofrece el resultado del riesgo cardiovascular con solo ingresar seis parámetros (nivel de colesterol total, cifra de presión arterial, padecer de diabetes mellitus, sexo, edad y tabaquismo) proporcionando un resultado de predicción de riesgo a 10 años de eventos cardiovasculares mayores 29,30 .

\section{SCORE}

La calculadora de Evaluación Sistematicadel Riesgo Coronario (SCORE), tiene como meta predecir el riesgo de mortalidad de causa cardiovascular a 10 años en individuos sin enfermedad previa. Se desarrolló en el año 2003, apoyándose en la base de datos de 12 poblaciones europeas y varios estudios de cohorte, con una población total de 205178 individuos. El modelo es aplicable a personas de entre 45 y 64 años, sin antecedentes de enfermedad cardiovascular y evalúa factores de riesgo como sexo, edad, tabaquismo, perfil lipídico y presión arterial sistólica. Estas variables contribuyen en el riesgo global de presentar un futuro evento trombo-isquémico ${ }^{31}$. Se fundamenta en el nivel de colesterol total o en la relación entre el colesterol total y el HDL, como variables independientes. Estas variables fueron calculadas para países europeos de bajo y alto riesgo por separado.

\section{Gaziano}

En el año 2008, Gaziano et al., colectaron datos por medio de la primera encuesta examinadora de salud y nutrición nacional de Estados Unidos, (NHANES I por sus siglas en inglés), para desarrollar una calculadora de riesgo de enfermedad cardiovascular, sin la necesidad de realizar pruebas de laboratorio. La novedad de este modelo es la similitud de puntaje que presenta al compararla con los modelos que requieren valores de laboratorio, la rapidez y simplicidad con la que se obtiene la puntuación, incluso desde la primera visita al consultorio y a un menor costo 32,33 .

Propusieron suplantar en el modelo de Framingham la medición de colesterol total y colesterol HDL y, en su lugar, utilizar el índice de masa corporal; además, implementaron factores como la edad (35-74 años), el sexo, la presión arterial sistólica, el tabaquismo y la diabetes mellitus.

Pandya et al. evaluaron la intercambiabilidad de la escala de Gaziano con respecto a escalas que utilizan pruebas de laboratorio, como Framingham en dos versiones (versiones 2008 y 1991) y con dos versiones de SCORE (para entornos de alto y bajo riesgo), que son generalmente usadas en la práctica clínica. Se hicieron comparaciones utilizando datos poblacionales de la tercera encuesta nacional examinadora de salud y nutrición de Estados Unidos (NHANES III), con resultados comparables entre las escalas.

Un enfoque preventivo y de educación en salud que empodere a los pacientes a seguir las recomendaciones de estilos de vida $y$, en caso de ser necesario, la adherencia al tratamiento, han sido la piedra angular para un adecuado control y en el mejor de los casos, tendencia a la baja de la mortalidad por eventos cardiovasculares en los países desarrollados ${ }^{34,35}$. Sin embargo, la situación en Latinoamérica se torna más difícil, debido a la deficiente educación con un enfoque preventivo de su población y la alta prevalencia de factores de riesgo cardiovascular.

Con el fin de evaluar el riesgo cardiovascular de la población latinoamericana se diseñó en el año 2003 el estudio la evaluación de riesgo cardiovascular múltiple en Latinoamérica (CARMELA por sus siglas en inglés) ${ }^{36}$, en siete ciudades de la región en dónde se evaluaron 11550 sujetos, de ambos sexos, de entre 25 y 64 años.

Este estudio proporcionó datos epidemiológicos sobre el riesgo de enfermedad cardiovascular, distribuido de forma heterogénea en Latinoamérica, con factores concentrados en diferentes ciudades. La prevalencia de hipertensión arterial fue $25 \%$ en Buenos Aires, Barquisimeto y Santiago, com- 
parables a la prevalencia mundial de $26 \%$ y Estados Unidos (con un 29 \%), según fecha de realización del estudio. México tuvo las mayores prevalencias de diabetes (9\%), síndrome metabólico (27\%) y obesidad (31\%), frente al resto de ciudades ${ }^{37}$. El riesgo cardiovascular general se determinó mediante la calculadora de riesgo de Framingham: $11 \%$ a $18 \%$ de la población de cada ciudad presentó un nivel de riesgo intermedio a alto ${ }^{38,39}$.

Al comparar datos de LASO y NHANES se evidenció que la prevalencia de hipertensión, diabetes y tabaquismo es similar en las poblaciones de Latinoamérica y Estados Unidos. Sin embargo, la obesidad y un valor alto de colesterol total es predominante en Estados Unidos, en contraste con la presencia de bajos niveles de colesterol HDL en Latinoamérica.

Uno de los principales problemas con respecto al cálculo del riesgo cardiovascular en la población de Latinoamérica, es que no se ha continuado investigando la evolución que experimentan los factores de riesgo en la población y subrogando la aplicación de las calculadoras de predicción de eventos cardiovasculares.

Para enfatizar la importancia del estado cardiovascular en las personas, la Asociación Americana del Corazón creó el concepto de Salud Cardiovascular Ideal; que se define como «la presencia simultánea de cuatro conductas cardiovasculares favorables: no fumar, IMC $<25 \mathrm{~kg} / \mathrm{m}^{2}$, actividad física y una dieta según las recomendaciones actuales; y tres factores de salud ideales: colesterol total $<200 \mathrm{mg} / \mathrm{dL}$, presión arterial $<120 /<80$ $\mathrm{mmHg}$ y glucosa en ayunas $<100 \mathrm{mg} / \mathrm{dL}$, todos estos valores sin necesidad de tratamiento médico» ${ }^{40,41}$.

Con el objetivo de evaluar la presencia de la Salud Cardiovascular Ideal en Latinoamérica, Seron et al., tomaron como población a los participantes del estudio CESCAS I (Detección y seguimiento de enfermedades cardiovasculares y factores de riesgo en el cono sur de Latinoamérica) desarrollado en 4 ciudades de Argentina, Chile y Uruguay, obteniendo como resultado que solo el 0,1\% de la población de estudio cumplió con los siete componentes de la definición.

En la actualidad se dispone de múltiples calculadoras de evaluación de riesgo cardiovascular. Cada modelo integra diferentes variables, con ciertos puntajes; por tanto se han realizado estudios para comparar cuál calculadora es más asertiva acorde a los eventos que se presentan y, sobre todo, cuáles serían las más adecuadas al contexto de la población donde son aplicadas ${ }^{42,43}$. Debido a la heterogeneidad de las poblaciones a nivel mundial, se ha intentado encontrar un método de predicción ideal, que sea extrapolables, así como diferencias entre hombres y mujeres en sus resultados (Tabla 1).

El modelo de Framingham se ha considerado un referente en la práctica clínica ${ }^{44}$. En algunas escalas, como SCORE, se ha cuestionado la cantidad de variables que evalúa. En ciertos casos podría sugerir que minimiza el riesgo obtenido, al no considerar factores como alteraciones en la glicemia o triglicéridos, el sedentarismo, índice de masa corporal o antecedentes familiares. Otra limitante es el aumento de costos en la atención médica, ya que exige exámenes de laboratorio ${ }^{45}$.

Tabla 1. Resultados de discriminación de riesgo para escalas basadas en exámenes de laboratorio en comparación con la escala de Gaziano

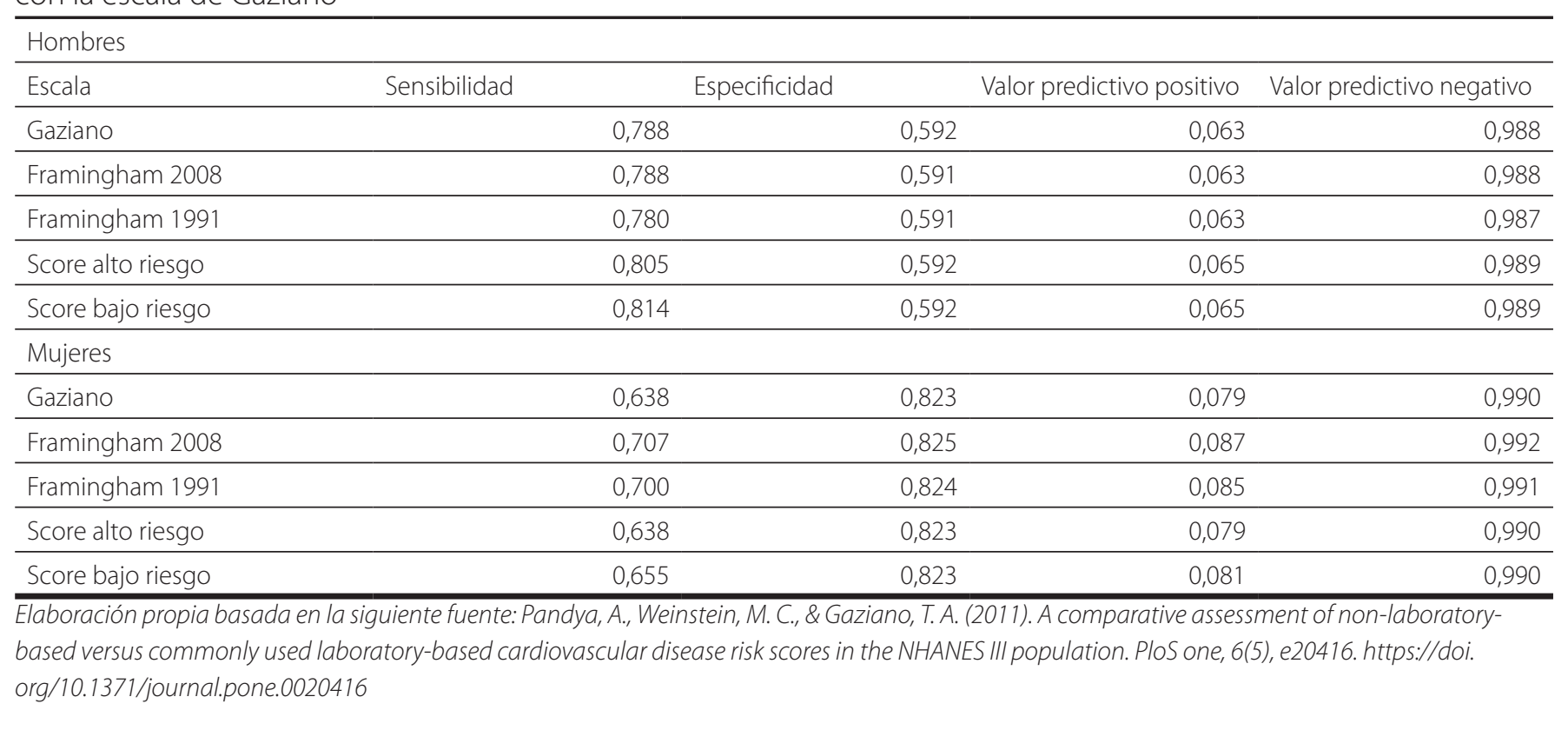


Muñoz et al., analizaron la concordancia entre las ecuaciones AHA/ACC 2013, SCORE y Framingham ajustado; evaluaron 800 pacientes colombianos entre 40 y 74 años. Los resultados evidenciaron que al utilizar la calculadora de Framingham ajustado había un 5,9\% de pacientes de alto riesgo; según SCORE para países de bajo riesgo un 18,7\% y para países de alto riesgo de 31,2 \%, existiendo una baja concordancia entre estos modelos. De acuerdo con la necesidad de administración de tratamiento hipolipemiante, la AHA/ACC lo estaría indicando a un 40,8\% de los pacientes, frente a un 50,6 \% según Framingham ajustado ${ }^{46,47}$.

El estudio realizado por Alcocer et al., en México comparó los métodos Framingham y SCORE, tomando la base de datos de un grupo de trabajadores del Hospital General, aparentemente sanos. Contrario a los resultados en Colombia, se observó que los pacientes en alto riesgo fueron mejor identificados con Framingham, mientras que SCORE los clasificó en riesgo medio y a algunos hasta en bajo riesgo, lo que sugiere que este modelo puede llegar a subestimar la presencia de enfermedad.

Peer et al., en el estudio CRIBSA, evaluaron la comparabilidad del porcentaje obtenido con la escala de Framingham versus la de Gaziano (NHANES I), en una población de Sudáfrica. Los resultados mostraron una alta correlación del puntaje entre la escala que utiliza exámenes de laboratorio y la que no los requiere. Así, se vuelve una alternativa factible al eliminar la necesidad de mediciones de laboratorio que incrementan el uso de recursos, mejorando la accesibilidad a un adecuado manejo de los pacientes en los países de bajos y medianos ingresos ${ }^{48}$.

Kariuki y colaboradores realizaron una búsqueda bibliográfica para evaluar la aplicabilidad de modelos que no necesitan valores de laboratorio. La investigación se centró en 5 escalas: Framingham no basado en laboratorio, Gaziano, OMS/ISH, método sueco basado en consultas y el modelo de práctica general del Reino Unido. Todos fueron desarrollados con el fin de ser usados en entornos de recursos limitados. El método sueco, Framingham y Gaziano demostraron una buena discriminación. Una ventaja de las escalas de Gaziano y de la OMS fue la simplicidad gráfica para su aplicación clínica ${ }^{49}$.

Pandya et al., realizaron un estudio para comparar la escala de Gaziano con la Escala de Framingham (1991-2008) y SCORE (alto y bajo riesgo), en una muestra de 5999 estadounidenses pertenecientes al NHANES III, que son individuos que también formaron parte del NHANES I, estudio donde se constituyó el modelo de Gaziano. Los re- sultados sugieren una sensibilidad, especificidad, valor predictivo positivo y negativo similares para todas las escalas (Tabla 1). Las variaciones más evidentes se presentan en la sensibilidad, marcadas por el sexo. Esta diferencia en el sexo femenino podría deberse a efectos biológicos diferenciales para variables específicas de cada escala, como el colesterol en la escala de Framingham o la diabetes y el tratamiento de la hipertensión en el modelo de SCORE. Esto se convierte en un punto de interés y requiere que futuras investigaciones evalúen si se presenta esta tendencia en otras poblaciones; y así valorar el uso de la escala de Gaziano como un modelo simple y de bajo coste para la atención temprana en la prevención de eventos isquémicos en países subdesarrollados.

A pesar de que estos estudios intentan encontrar la mejor calculadora de riesgo para la población latinoamericana, aún no se dispone de un modelo definitivo. Por tanto, es de esperar que esto influya en que, a la fecha, no se logran alcanzar las metas establecidas en las estrategias de prevención. La identificación oportuna del riesgo en los individuos resulta en una mejoría de los resultados clínicos de morbimortalidad y se puede convertir en el punto de partida para adecuar políticas de decisiones pertinentes sobre prevención y control de eventos cardiovasculares ${ }^{50}$.

\section{Conclusiones}

Han sido expuestas múltiples calculadoras para predecir el riesgo cardiovascular, que se diferencian entre sí en los criterios que incluyen para realizar su cálculo y el evento que están destinadas a predecir. Se ha comprobado su efectividad y utilidad para prevenir las formas graves de enfermedades cardiovasculares principalmente en el primer nivel de atención en salud.

Al describir las escalas de Framingham, el algoritmo QRISK3, la calculadora de ACC/ AHA, OMS, el SCORE y la propuesta de Gaziano, se evidencia cuáles variables coinciden como edad, género, colesterol total, valor de presión arterial sistólica, diabetes mellitus y tabaquismo. El tiempo de estimación de riesgo es, en su mayoría, para 10 años.

Para calcular el riesgo cardiovascular se requiere el uso de exámenes de laboratorio, una considerable limitación principalmente en países en desarrollo. Debido a esto, el modelo propuesto por Gaziano, que no incluye exámenes de laboratorio, es una prometedora elección con igual capacidad predictiva respecto a otras que sí los incluyen. Sin embargo, todavía se necesitan estudios de validación externa que confirmen 
su utilidad y eficacia en población de países latinoamericanos.

\section{Agradecimiento}

A nuestras familias por el apoyo desde la idea del tema, a nuestro asesor que siempre nos dio ánimos para continuar y sugiriendo correcciones para presentar un mejor trabajo.

\section{Financiamiento}

El estudio fue financiado por cada autor.

\section{Referencias bibliográficas}

1. Brotons C, Moral I, Fernández D, Puig M, Calvo Bonacho E, Martínez Muñoz P, et al. Estimación del riesgo cardiovascular de por vida (IBERLIFERISK): una herramienta nueva en prevención primaria de las enfermedades cardiovasculares. Rev Esp Cardiol. 2019;72(7):562-568. DOI: 10.1016/j. recesp.2018.05.002

1. Sánchez-Arias AG, Bobadilla-Serrano ME, Dimas-Altamirano B, Gómez-Ortega M, González-González G. Enfermedad cardiovascular: primera causa de morbilidad en un hospital de tercer nivel. Rev Mex Cardiol. 2016;27(S3):98-102. Disponible en: https://www.medigraphic.com/cgi-bin/ $\underline{\text { new/resumen.cgi?IDARTICULO }=66578}$

2. Saturno D, Castillo M, Brea K, Figueroa F, Medina H, \& Moreno N. Enfermedad cardiovascular y obesidad. Revista Vive. 2018;1 (1):59-67. DOl:10.33996/revistavive. v111.5

3. Velásquez J, Guerrero F, Estacio M, Vergara I, Yara S, Carrillo D. Incidencia de eventos cardiovasculares mayores asociada a la suspensión de doble terapia antiagregante en pacientes con enfermedad coronaria e implante de stent. Rev Colomb Cardiol. 2017;24(4):342-50. DOI: 10.1016/j. rccar.2016.10.038

4. Nuñez G, López I, Ramos S, Ramos E, Guevara M, González I. Riesgo cardiovascular en pacientes de primer nivel de atención. Rev Salud Publica Nutr. 2015;14(1):1-8. Disponible en: http://respyn.uanl.mx/index. php/respyn/article/view/1

5. Pereira J, Peñaranda D, Reyes A, Cáceres K, Cañizarez Y. Prevalencia de factores de riesgo cardiovascular en América Latina: una revisión de la evidencia publicada de 2010 a 2015. Rev.Mex.Cardiol. 2015;26(3):125-39. Disponible en: http://www.scielo.org.mx/

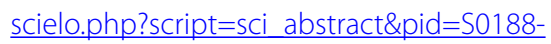
21982015000300004\&lng=es\&nrm=iso\&t| $\underline{n g}=\mathrm{es}$

6. Ochoa A, García D. Estimación del riesgo enfermedad cardiovascular en el contexto de la Atención Primaria de Salud. Cuba y Salud. 2016;11(1):47-57. Disponible en: https://www.medigraphic.com/cgi-bin/ new/resumen.cgi?IDARTICULO $=66970$

7. Achiong M, Achiong F, Afonso de León J, Álvarez M, Suárez M. Riesgo cardiovascular global y edad vascular: herramientas claves en la prevención de enfermedades cardiovasculares. Rev. Med. Electrón. 2016;38(2):211-26. Disponible en: http:// scielo.sld.cu/scielo.php?script=sci abstract\& pid=S168418242016000200010\&lng=es\&nr $\underline{m=i s o \& t \operatorname{lng}=e s}$

8. Sarre D, Cabrera R, Rodríguez F, Díaz E. Atherosclerotic cardiovascular disease. Review of risk scales and cardiovascular age. Med. Int. Mex. 2018;34(6):910-923. DOI: 10.24245/mim.v34i6.2136.

9. Veliz L, Mendoza S, Barriga O. Adherencia terapéutica y control de los factores de riesgo cardiovasculares en usuarios de atención primaria. Enfermería Universitaria. 2015;12(1):3-11. DOI: 10.1016/j. reu.2015.05.003

10. Vicente B, Vicente E, Costa M. Estimation of cardiovascular risk in patients with type 2 diabetes. Finlay. 2015;5(3):178-89. Disponible en: http://www.revfinlay.sld.cu/ index.php/finlay/article/view/276

11. Carrillo R, Altez C, Pacheco N, Bambs C, Irazola V, Miranda JJ, et al. Cardiovascular Disease Prognostic Models in Latin America and the Caribbean. Glob Heart. 2019;14(1):81-93. DOI: 10.1016/j. gheart.2019.03.001

12. Núñez R, Lopez E, Hernández R, Peña R, Guevara M, González I. Riesgo cardiovascular en pacientes de primer nivel de atención. Rev Salud Publica Nutr. 2015;14(1):1-8. Disponible en: http://respyn.uanl.mx/index. php/respyn/article/viewFile/1/1

13. Brotons C, Moral I, Fernández D, Cuixart L, Muñoz A, Soteras A, et al. Clinical consequences of using the new cardiovascular risk tables SCORE OP in patients aged over 65 years. Med Clín (Barc.). 2016;147(9):381-386. DOI: 10.1016/j. medcli.2016.06.035.

14. Almonacid C, Camarillo M del S, Gil Z, Medina C, Rebellón J, Mendieta H. Evaluación de factores de riesgo asociados a enfermedad cardiovascular en jóvenes universitarios de la Localidad Santafé en Bogotá, Colombia. NOVA publ cient. 2016;14(25):35-45. Disponible en: http:// www.scielo.org.co/scielo.php?script=sci art text\&pid $=S 1794-24702016000100004$

15. Tsao C, Vasan R. Cohort Profile: The Framingham Heart Study (FHS): overview of milestones in cardiovascular epidemiology. International journal of epidemiology. 2015;44(6):1800-1813. DOI:10.1093/ije/ dyv337 
16. Manson J, Bassuk S. Invented commentary: The Framingham Offspring Study-A Pioneering investigation into familial aggregation of cardiovascular risck. Am J Epidemiol. 2017; 185(11):1103-1108. DOI: 10.1093/aje/kwx068

17. Govindaraju D, Adrienne L, Kannel W, O'Donnell C, Atwood L, D'Agostino R, et al. Genetics of the Framingham Heart Study population. Advances in genetics. 2008;62:33-65. DOI: 10.1016/S00652660(08)00602-0

18. Zheng S, Lubin B, Au R, Murabito JM, Benjamin EJ, Shwartz M. Advantages of Continuous-Valued Risk Scores for Predicting Long-Term Costs: The Framingham Coronary Heart Disease 10-Year Risk Score. Adv Geriatr Med Res. 2019;1(1):e190004. DOI: 10.20900/ agmr20190004

19. Mahmood S, Levy $D$, Vasan R, \& Wang T. The Framingham Heart Study and the epidemiology of cardiovascular disease: a historical perspective. Lancet. 2014;383(9921):999-1008. DOI: 10.1016/ S0140-6736(13)61752-3

20. Hippisley J, Coupland C, Brindle P. Development and validation of QRISK3 risk prediction algorithms to estimate future risk of cardiovascular disease: prospective cohort study. BMJ. 2017;357:j2099. DOI: 10.1136/bmj.j2099

21. Bandyopadhyay S, Wolfson J, Vock D, Vázquez G, Adomavicius G, Elidrisi M, et al. Data mining for censored time-toevent data: a Bayesian network model for predicting cardiovascular risk from electronic health record data. Data Min Knowl Disc. 2015;29(4):1033-69. Disponible en: DOI:10.1007/s10618-014-0386-6

22. Unnikrishnan P, Kumar D, Poosapadi S, Kumar H, Mitchell P, Kawasaki R. Development of Health Parameter Model for Risk Prediction of CVD Using SVM. Computational and Mathematical Methods in Medicine. 2016. DOI: 10.1155/2016/3016245

23. Alageel S, Wright AJ, Gulliford M. Changes in cardiovascular disease risk and behavioural risk factors before the introduction of a health check programme in England. Preventive Medicine. 2016;91:158-63. DOI: 10.1016/j.ypmed.2016.08.025

24. Lloyd D, Braun L, Ndumele C, Smith S, Sperling L, Virani S, et al. Use of Risk Assessment Tools to Guide Decision-Making in the Primary Prevention of Atherosclerotic Cardiovascular Disease: A Special Report From the American Heart Association and American College of Cardiology. 2019;139(25): e1162-77. DOI: 10.1161/ CIR.0000000000000638

25. Arnett K, Blumenthal S, Albert A, Buroker B, Goldberger D, Hahn J, et al. 2019 ACC/
AHA Guideline on the Primary Prevention of Cardiovascular Disease: A Report of the American College of Cardiology/ American Heart Association Task Force on Clinical Practice Guidelines. Circulation. 2019;140(11):e596-646. DOI: 10.1161/ CIR.0000000000000678

26. Sánchez M de LP, Rodríguez MA, Cruz RG. Estimación del riesgo cardiovascular en una población del área de salud del Policlínico Santa Clara. Medicentro Electrónica. 2016;20(1):38-45-45. Disponible en: http://scielo.sld.cu/scielo. php?script=sci arttext\&pid=S102930432016000100006\&lng=es.

27. Rojas NBA, Herrera AD, Medina RS, Rojas LL, García R de la N, Pérez PV, et al. Estimación del Riesgo Cardiovascular Global en el Municipio Colón. Matanzas, Cuba. Revista Cubana de Cardiología y Cirugía Cardiovascular. 2016;22(3):134-42. Disponible en: http://www.revcardiologia. sld.cu/index.php/revcardiologia/article/ view/656

28. Prieto AF, Gaglio R, Monsalvo M, Rezzonico G, Galli A, Cerezo L. Estudio cualitativo: uso de la guía para la estimación del riesgo cardiovascular global en la práctica clínica. Revista de Salud Pública. 2015;19(2):42-53. DOI: 10.1016/S1131-3587(11)15002-5

29. Hernández Vázquez $L$, de la Vega Pazitková T, Pérez Martínez V, González Delgado E. Riesgo cardiovascular en pacientes de un consultorio médico del policlínico "Ana Betancourt". Rev Cubana Med Gen Integr. 2012;28(4):569-584. Disponible en: http://scielo.sld.cu/scielo. php?script=sci arttext\&pid=S086421252012000400002\&lng=es.

30. Baena-Díez JM, Subirana I, Ramos R, Gómez de la Cámara A, Elosua R, Vila J, et al. Evaluación de la validez de las funciones SCORE de bajo riesgo y calibrada para población española en las cohortes FRESCO. Rev Esp Cardiol. 2018;71(4):274-82. DOI: 10.1016/j.recesp.2017.03.016

31. Pandya A, Weinstein MC, Gaziano TA. A comparative assessment of non-laboratorybased versus commonly used laboratorybased cardiovascular disease risk scores in the NHANES III population. PloS one. 2011;6(5), e20416. DOI 10.1371/journal. pone.0020416

32. Rojas NBA, Herrera AD, Medina RS, Rojas LL, García R de la N, Pérez PV, et al. Estimación del Riesgo Cardiovascular Global en el Municipio Colon. Matanzas, Cuba. Revista Cubana de Cardiología y Cirugía Cardiovascular. 2016;22(3):134-42. Disponible en: http://www.revcardiologia. sld.cu/index.php/revcardiologia/article/ view/656 
33. Alcalá López JE, Maicas Bellido C, Hernández Simón P, Rodríguez Padial L. Cardiopatía isquémica: concepto, clasificación, epidemiología, factores de riesgo, pronóstico y prevención. Medicine Programa de Formación Médica Continuada Acreditado. 2017;12(36):2145-52. DOI: 10.1016/j.med.2017.06.010

34. Pereira-Rodríguez J, Peñaranda-Florez D, Reyes-Sáenz A, Cáceres-Arévalo K, CañizarezPérez Y. Prevalence of cardiovascular risk factors in Latin America: a review of the published evidence 2010-2015. Revista mexicana de cardiología. 2015;26(3):125-39. Disponible en: http://www.scielo.org.mx/ scielo.php?script $=$ sci abstract\&pid=S018821982015000300004\&lng=es\&nrm=iso\&t| ng=es

35. Garza-López EP, Silva-Ruiz R, RodríguezPérez CV. Factores de riesgo cardiovascular en pacientes con diabetes mellitus tipo 2 atendidos en consulta externa. Salud Pública de México. 2017;59(6):604-5. DOI: 10.21149/8511

36. Touboul P-J, Vicaut E, Labreuche J, Acevedo M, Torres V, Ramírez-Martínez J, et al. Common Carotid Artery IntimaMedia Thickness: The Cardiovascular Risk Factor Multiple Evaluation in Latin America (CARMELA) Study Results. CED. 2011;31(1):43-50. DOI: 10.1159/000320264

37. Miranda JJ, Herrera VM, Chirinos JA, Gómez LF, Perel P, Pichardo R, et al. Major Cardiovascular Risk Factors in Latin America: A Comparison with the United States. The Latin American Consortium of Studies in Obesity (LASO). PLOS ONE. 2013;8(1):e54056. DOI: 10.1371/journal.pone.0054056

38. Álvarez-Ceballos JC, Álvarez-Múñoz AM, Carvajal-Gutiérrez W, González MM, Duque JL, Nieto-Cárdenas OA. Determinación del riesgo cardiovascular en una población. Revista Colombiana de Cardiología. 2017;124(4):334-41. DOI: 10.1016/j. rccar.2016.08.002

39. Lloyd-Jones DM, Hong Y, Labarthe $D$, Mozaffarian D, Appel LJ, Van Horn L, et al. Defining and Setting National Goals for Cardiovascular Health Promotion and Disease Reduction: The American Heart Association's Strategic Impact Goal Through 2020 and Beyond. Circulation. 2010;121(4):586-613. DOI: 10.1161/ CIRCULATIONAHA.109.192703

40. Seron P, Irazola V, Rubinstein A, Calandrelli M, Ponzo J, Olivera $\mathrm{H}$, et al. Ideal Cardiovascular Health in the southern cone of Latin America. Public Health. 2018;156:132-9. DOI: 10.1016/j.puhe.2017.12.017

41. Agüero AO, Duménigo GG. Estimación del riesgo enfermedad cardiovascular en el contexto de la Atención Primaria de Salud. Cuba y Salud. 2016;11(1):47-57. Disponible en: http://www.revpanorama.sld.cu/index. php/panorama/article/view/484

42. Alegría Ezquerra E, Alegría Barrero A, Alegría Barrero E. Estratificación del riesgo cardiovascular: importancia y aplicaciones. Rev Esp Cardiol. 2012 Jun;12:8-11. DOl: 10.1016/S1131-3587(12)70039-0

43. Rodríguez AA, Murillo AA, Rivera RJ, Montalván EE, Duarte KG, Urrutia SA, et al. Validez de los métodos de predicción para riesgo cardiovascular en América Latina: revisión bibliográfica. Rev méd hondur. 2017;85(1-2):51-55. Disponible en: http:// www.bvs.hn/RMH/pdf/2017/pdf/Nol85-1-22017-15.pdf

44. TomasikT, Krzysztoń J, Dubas-Jakóbczyk K, Kijowska V, Windak A. The systematic coronary risk evaluation (SCORE) for the prevention of cardiovascular diseases. Does evidence exist for its effectiveness? A systematic review. Acta cardiológica.2017;72(4), 370-379. DOI: 10.1080/00015385.2017.1335052

45. Muñoz V OM, Ruiz Morales ÁJ, Mariño Correa A, Bustos C. MM. Concordancia entre los modelos de SCORE y Framingham y las ecuaciones AHA/ACC como evaluadores de riesgo cardiovascular. Revista Colombiana de Cardiología. 2017;24(2):110-6. DOI: 10.1016/j.rccar.2016.06.013

46. Alcocer LA, Lozada O, Fanghänel G, Sánchez Reyes L, Campos Franco E. Estratificación del riesgo cardiovascular global. Comparación de los métodos Framingham y SCORE en población mexicana del estudio PRIT. Cirugía y cirujanos. 2011;79(2):168-74. Disponible en: https://biblat.unam.mx/ es/revista/cirugia-y-cirujanos/articulo/ estratificacion-del-riesgo-cardiovascularglobal-comparacion-de-los-metodosframingham-y-score-en-poblacionmexicana-del-estudio-prit

47. Gaziano TA, Pandya A, Steyn K, et al. Comparative assessment of absolute cardiovascular disease risk characterization from non-laboratory-based risk assessment in South African populations. BMC Med.2013;11:170. DOI: 10.1186/1741-701511-170

48. Kariuki JK, Stuart-Shor EM, Leveille SG, Hayman LL. Evaluation of the performance of existing non-laboratory based cardiovascular risk assessment algorithms. BMC Cardiovasc Disord.2013;13:123. DOI: 0.1186/1471-2261-13-123

49. Motamed N, Rabiee B, Perumal D, Poustchi H, Miresmail SJH, Farahani B, et al. Comparison of cardiovascular risk assessment tools and their guidelines in evaluation of 10-year CVD risk and preventive recommendations: A population based study. International Journal of Cardiology.2017;228:52-7. DOI: 10.1016/j.ijcard.2016.11.048 